\title{
Solution of a high bridge pillar under wind effects taking into account the real properties of reinforced concrete
}

\author{
Katarína Tvrdá ${ }^{1, *}$ \\ ${ }^{1}$ Slovak Technical University in Bratislava, Faculty of Civil Engineering, Department of Structural \\ Mechanics, Radlinskeho 11, 81005 Bratislava, Slovak Republic
}

\begin{abstract}
The current standard is that the calculation of a reinforced concrete structure is carried out using the theory of elasticity and its design is based on the requirements of EC2. In this paper, a computational method based on direct numerical integration and Gaussian or Newton-Cotes cubicles that directly reflect the material properties determined by EC2 is presented. The deviations in the internal forces and deformations achieved compared to the classical theory of elasticity are demonstrated in the calculation of the high bridge pillar.
\end{abstract}

\section{Introduction}

Presently built bridge structures are still taller and slimmer, on the other hand, due to the incorporation of complementary structures such as windbreaks or noise barriers, increasing horizontal loads of these structures should be considered. This implies an increase in horizontal deformations and a greater influence of the second order theory on the resulting deformation of the structure.

The calculation of the influence of the second order theory of compression bars is traditionally made on the basis of the simplification assumptions under point 5.8.5 of Eurocode 2 [1], where the non-linear calculation is replaced by a linear, adjusted input data and correction coefficients, which take into account the non-linear properties of the materials only to some extent (see Eurocode 2 articles 5.8.7 and 5.8.8). In complicated cases, non-linear analysis is carried out using standard FEM programs [2], which, however, depending on the specific program, work with simplifying design models (blurry reinforcement, concrete tensile strength, polygonal stress behaviour in concrete, etc.).

This deficiency is solved in the presented work, in which we present a completely new method of direct non-linear calculation of deformation of beam structures, taking into account physical and geometric non-linearity while maintaining exactly the required properties of materials.

\footnotetext{
*Corresponding author: katarina.tvrda@stuba.sk
} 


\section{Non-linear material properties as defined by the Eurocode and the resulting consequences}

The working diagram for non-linear static analysis is defined by Eurocode 2, Art. 3.1.5. Design working diagram of steel as defined in article 3.2.7. Based on Article 6.1, we can consider planar deformation of the cross-section, Fig. 1.

By applying these three clauses of the standard to the calculation, a single possible cross-section deformation $\varepsilon_{j}$ can be unambiguously assigned to each defined geometry and cross-section material and to each external load.

This defined a single value of cross-section rotation $\kappa_{j}\left(M, N, w_{x}, x\right)$ as a reaction of the differential cross-section of the structure to the external load.

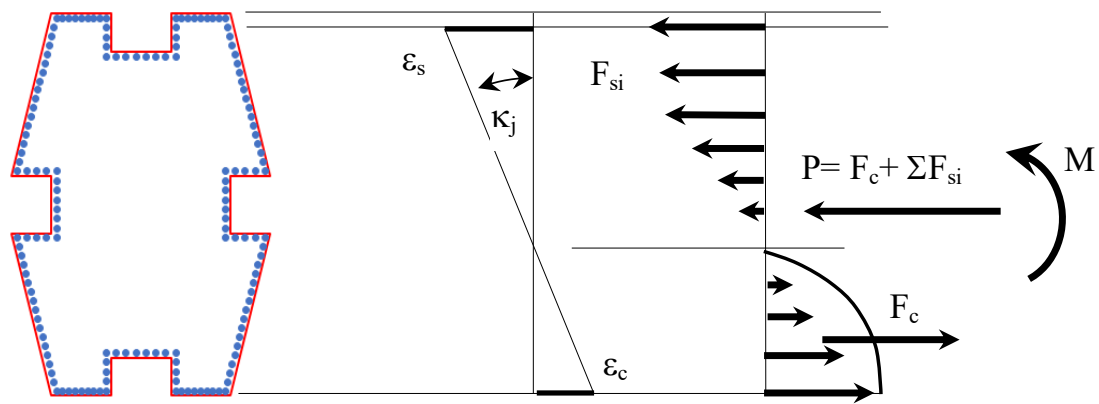

Fig. 1. Characteristics in $j$-section.

If we repeat this calculation for the given parameters (geometry, material, normal force) and gradually change the deformation values within the standard-defined boundaries, we get a discrete dependency of the cross-sectional rotation $\kappa_{j}$ from the external load, which using any form of interpolation, can be easily adjusted to a functional dependence $M$ and $\kappa\left(M, N, w_{x}, x\right)$ with an accuracy dependent on the division density (see Fig. 2). The ultimate values of the moments are also the limit values of the bending capacity of the cross-section at the specified normal force.

\section{Calculation of deformation}

With the known functional dependence $M$ and $\kappa\left(M, N, w_{x}, x\right)$, the last task remains to obtain the resulting value of deformation from the known moment and curvature. Since the deformation methods do not work in this case, the superposition of effects does not apply either, we have to implement the basic equations of mechanics and we use the method of direct integration of the bending curve of the beam [3] (literally stack the deformed elementary cross-sections). We start from the basic integral equation for the deformation calculation taking into account the geometric non-linearity

$$
w(x)=\int_{0}^{x} \int_{0}^{x} \frac{M(x)+F \cdot(w(L)-w(x))}{E I\left(M, N, w_{x}, x\right)} d x d x=\int_{0}^{x} \int_{0}^{x} \kappa\left(M, N, w_{x}, x\right) d x d x
$$

where we changed expression (2) to a functional dependence of curvature 


$$
\kappa\left(M, N, w_{x}, x\right)=\frac{M(x)+F \cdot(w(L)-w(x))}{E I\left(M, N, w_{x}, x\right)} .
$$

Although the functional dependence $\kappa\left(M, N, w_{x}, x\right)$ can be considered as a function, its analytical description is so complicated and differentiated into individual parts that integration can be realized only numerically in discrete points (Fig. 3). At these discrete points, the values of $N$ and $x$ are constant and $\mathrm{w}_{\mathrm{x}}$ can be considered quasi-constant (constant for the individual calculation of the iterative cycle) and therefore the calculation is based exclusively on the curvature versus moment.

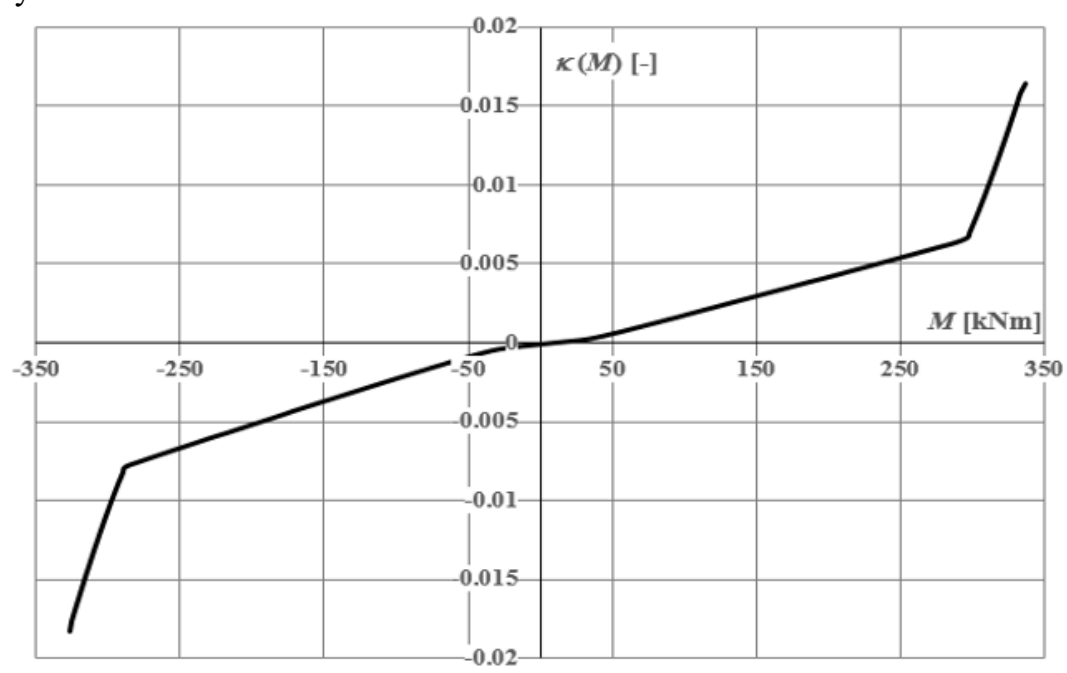

Fig. 2. Example of functional dependence $M$ and $\kappa(M)$.

\section{Method of numerical integration of repeated integrals}

The method of transformation of repeated integral to simpler integrals and the method of solving repeated integrals by extending Gaussian-type quadrature to cubes were published in the paper [4-6]. Given that such a calculation provides the resulting value of deformation only at the end of the length interval, in this work we decided to use for numerical integration of the Newton-Cotes type, which can be extended to cubic calculations by a similar methodology.

$$
\begin{gathered}
\underbrace{\int_{a} \int_{0}^{t} \int_{0}^{t} \cdots \int_{0}^{t} f}_{n} f(t) d t^{n}=\sum_{i=1}^{n-1} \frac{(b-a)^{i}}{i !} \int_{0}^{a} \int_{0}^{x_{1}} \cdots \int_{0}^{x_{n-i-1}} f\left(x_{n-i}\right) d x_{n-i} \cdots d x_{1}+ \\
\frac{1}{(n-1) !} \int_{a}^{b}(b-t)^{n-1} f(t) d t
\end{gathered}
$$

It follows from the boundary condition of the cantilever that the first integral of formula (3) completely falls out and the second integral of formula (3) is sufficient to calculate the deformation of the structure at non-linear physical properties. For its solution we can use a simple scheme, for example of the sixth degree of the numerical cubic with 6th polynomial accuracy, to which the formula applies 


$$
w(7)=\frac{3}{70} H^{2}\left(41 \kappa_{1}+180 \kappa_{2}+18 \kappa_{3}+138 \kappa_{4}+9 \kappa_{5}+36 \kappa_{6}\right),
$$

where $H$ is the distance between section (Fig. 3).

This short formula represents a numerical solution of the integral (1) for the cantilever. To calculate the second order theory, we will of course also need intermediate values of deformation $w\{1,2,3,4,5,6\}$ to take into account the influence of normal force on deformation. For their calculation we use a similar calculation scheme as in (4), calculated by integration of the Lagrange polynomial at the corresponding reduced interval.

\section{Procedure of calculation of vertical pillar deformation taking into account the second order theory}

The analysed pillar is divided along its height into $n$ equidistant pieces and a constant normal force is determined in each cross-section of the pillar, while normal force may be considered different in each cross-section.

Based on the given normal force, a discrete dependence $\kappa_{j}$ of variable moment is calculated at specific discrete points of the column, for all real deformations of the crosssection according to the standard.

The actual deformation of the pillar due to the horizontal forces is calculated by substituting the respective curvature from the known moment into the formula (4). If the second order theory is taken into account, the moment is then modified by the influence of the normal force on the deformed cross-section and the deformation is re-calculated.

The process of calculation of deformation and increase of bending moment by the influence of normal force is repeated until the required accuracy is achieved.

\section{Solution of the bridge pillar for wind effects}

The above described process was applied to the calculation of the bridge pillar deformation due to the horizontal load, which in our case represents the wind. Our work is based on the parameters of one existing bridge in Slovakia. The pillar is $32 \mathrm{~m}$ high and is loaded with vertical force $N=33000 \mathrm{kN}$.
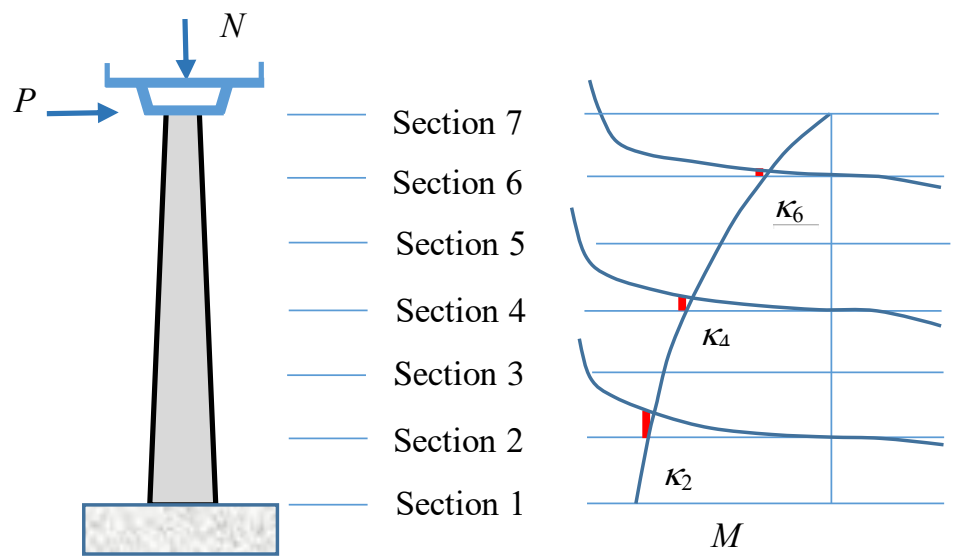

Fig. 3. Bridge pillar. 
Its cross-section decreases conically along the height of the pillar (Fig. 3). The pillar is reinforced over the entire height with the same number of 160 reinforcements $\Phi 28$ (Fig. 4), only the distance between them changes in height. The cross-section of the reinforced concrete pillar with the actual reinforcement distribution in sections 1 and 7 is shown in Fig. 4.

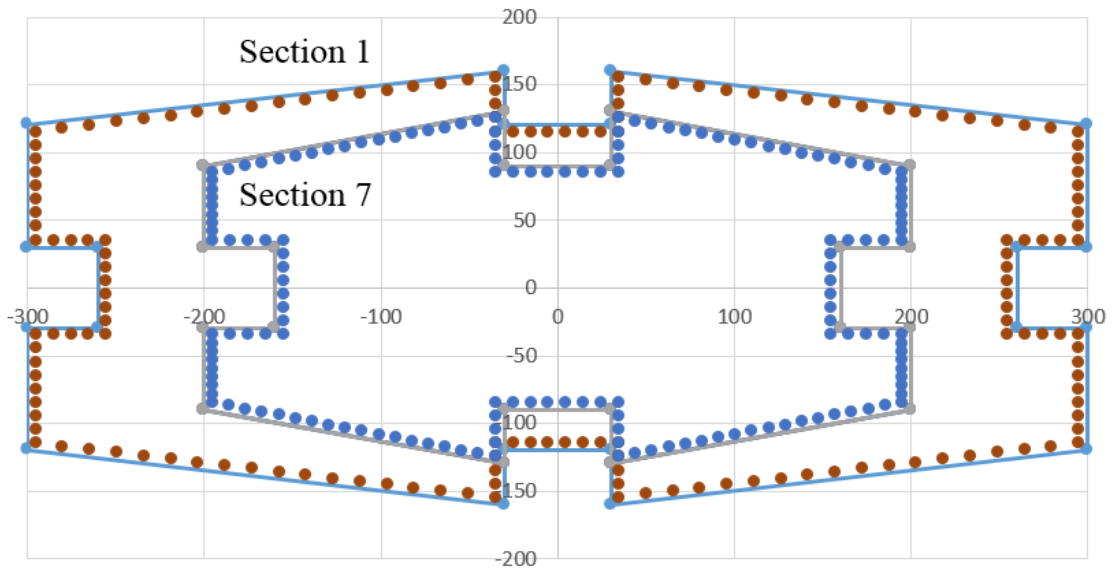

Fig. 4. Cross-section of reinforced concrete pillar in sections 1 and 7.

The curvature pattern in individual sections 1 to 7 is shown in Fig. 5.

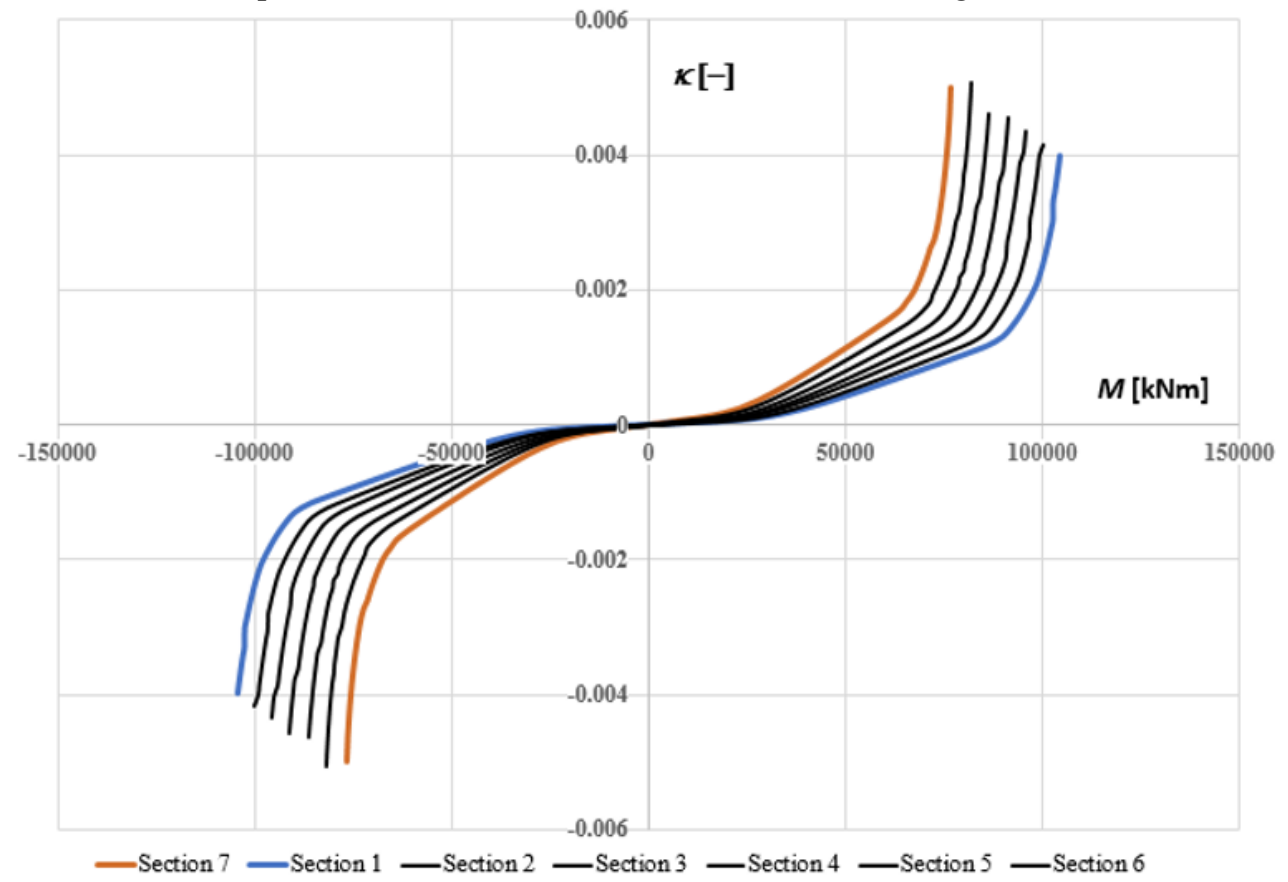

Fig. 5. Functional dependence of $M$ and $\kappa(M)$ at discrete points of the pillar in sections 1 and 7.

The course of the dependence of the horizontal force $P$ on the deformation $w$ without / with consideration of the second order theory is shown in Fig. 6. For comparison, there is also shown a linear solution without and with the second order theory. 


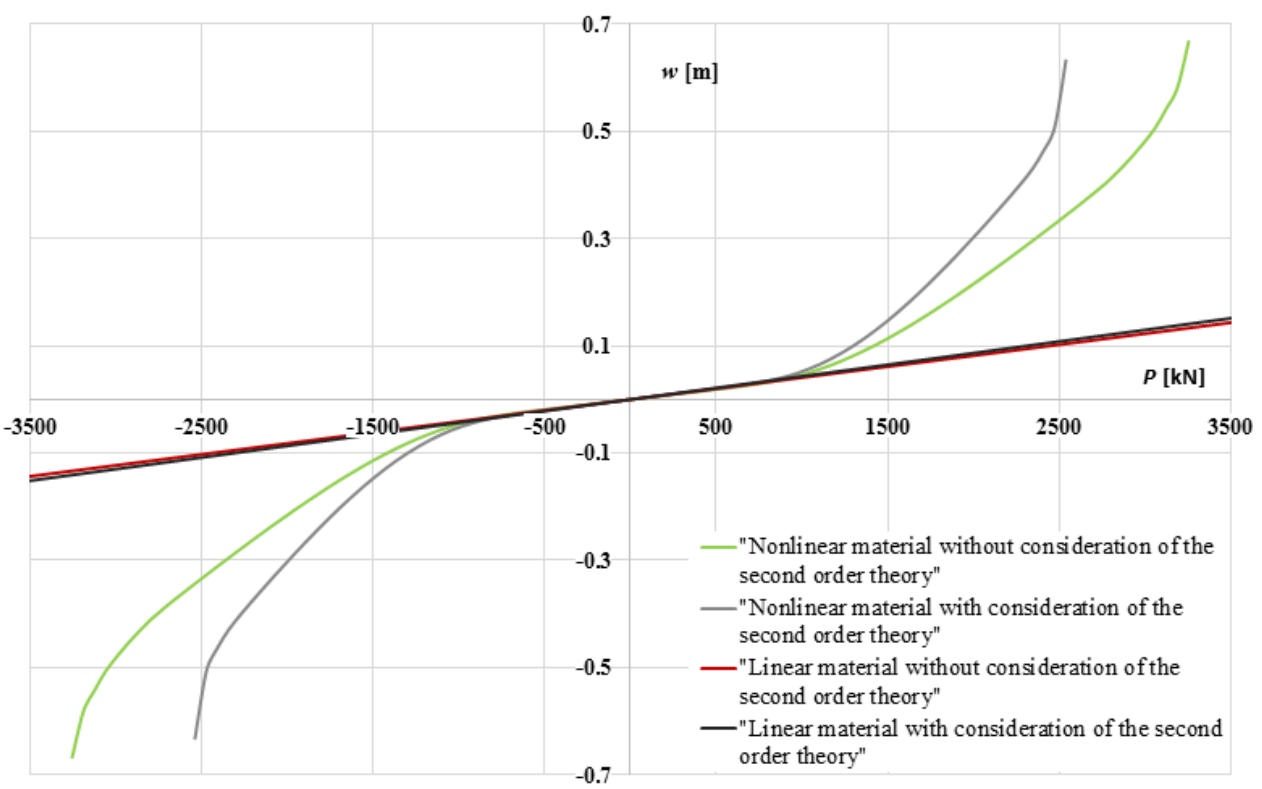

Fig. 6. Functional dependence of wind force $P$ and $w$ in section 7 .

The calculated horizontal wind load on this pillar is approximately $820 \mathrm{kN}$, which is exactly in the point, where the linear and non-linear branches diverge - the pillar satisfies.

Moreover, the presented calculation did not take into account the tension interaction of the concrete between the cracks in any way, thus showing slightly higher deformations than would be expected under real action.

\section{Conclusion}

The presented new method of direct numerical integration can easily calculate the deflection of a general reinforced concrete beam taking into account all common physical and geometric nonlinearities. The accuracy of the method can be influenced by the choice of the dividing density depending on the curvature and moment, as well as the pillar and the degree of the numerical cubature. In practice, however, the accuracy of the resulting deformation depends primarily on the material properties entered.

The paper has been supported by the Grant Agency SR, VEGA No. 1/0412/18.

\section{References}

1. STN EN 1992-2 Eurocode 2: Design of concrete structures

2. Ansys ${ }^{\circledR}$ User's Manual, Swanson Analysis Systems, Inc.

3. J. Dický, Z. Mistríková, J. Sumec, Elasticity and Plasticity in Civil Engineering (Slovak University of Technology, 2005)

4. K. Tvrdá, M. Minárová, Computation of Definite Integral over Repeated Integral. Tatra Mountains Mathematical Publications 75, 1-14 (2018), DOI:10.2478/tmmp-2018-0000

5. G. B. Folland, Advanced Calculus (Prentice Hall, 2002)

6. M. Abramowitz, I. Stegun, Handbook of Mathematical functions (Dover Publications, Inc. New York, 1972) 